\title{
Prevalência das lesões intra-epiteliais de alto grau em pacientes com citologia com diagnóstico persistente de ASCUS
}

\author{
Prevalence of high-grade squamous intraepithelial lesions in patients with persistent cytological \\ diagnosis of ASCUS
}

\author{
Fernanda Rangel da Veiga ${ }^{1}$, Fábio Russomano², Maria José de Camargo ${ }^{3}$, \\ Aparecida Cristina Sampaio Monteiro ${ }^{4}$, Aldo Reis ${ }^{5}$, Maria Aparecida Tristão ${ }^{6}$
}

\section{RESUMO}

Objetivo: verificar a prevalência de lesões intra-epiteliais de alto grau (LIAG) e câncer invasor em mulheres com citologia com diagnóstico de ASCUS (atipias em células escamosas de significado indeterminado) persistente após 6 meses e verificar se a idade é fator indicador para a existência destas lesões neste grupo de mulheres. Métodos: foram incluídos 215 casos de mulheres nãogestantes e HIV-negativas com diagnóstico de ASCUS (sem especificação) persistente em duas citologias com intervalo mínimo de 6 meses. Tais resultados foram confrontados com o resultado histológico de biópsias, exéreses da zona de transformação (large loop excision of the transformation zone) ou cones. Foram considerados negativos para LIAG ou câncer quando a colposcopia foi satisfatória e sem alterações ou quando, apesar de insatisfatória, não foi detectada lesão em pelo menos um seguimento citológico e colposcópico. Para estabelecer a prevalência de lesões, calculamos a freqüência de diagnósticos com seus respectivos intervalos de confiança a 95\% (IC 95\%). Para análise estatística da diferença de proporções de LIAG ou câncer em cada faixa de idade, foi utilizado teste do $\chi^{2}$,e ainda estimamos o risco destas lesões entre mulheres com mais de 35 anos pela razão de prevalências com seu IC $95 \%$. Resultados: encontramos um total de negativos de 49,3\% dos casos (IC 95\%: 42,6-55,9). A prevalência de lesões intra-epiteliais de baixo grau foi de 38,6\% (IC 95\%: 32,1-45,1) e de LIAG de 10,7\% (IC 95\%: 6,5-14,8). Casos de câncer foram encontrados em 1,4\% das pacientes (IC 95\%: 0-2,9). Não foi possível estabelecer, de forma significativa, maior risco de LIAG/câncer considerando o corte de idade em 35 anos. Conclusão: a prevalência de LIAG/câncer encontrada em nosso estudo mostra que o risco de encontrarmos este tipo de lesão em mulheres atendidas no Sistema Único de Saúde em nosso município com duas citologias com diagnóstico de ASCUS é de cerca de $12 \%$. Não foi possível evidenciar maior probabilidade de LIAG/câncer em qualquer das faixas etárias analisadas, porém este resultado pode ter sido limitado pelo pequeno tamanho amostral.

PALAVRAS-CHAVE: Células epidermóides atípicas; Neoplasia intra-epitelial cervical; Neoplasia do colo uterino; Câncer cervical; Colposcopia; Citodiagnóstico

\section{ABSTRACT}

Purpose: to determine the prevalence of high-grade squamous intraepithelial lesions (HSIL) and cancer in women with cytological diagnosis of persistent ASCUS (atypical squamous cells of undetermined significance) for 6 months in the last 7 years. We also assessed if age could be a predictive factor for presence of HSIL/cancer in this group. Methods: we included 215 cases of nonpregnant and HIV-seronegative women with cytological diagnosis of persistent ASCUS (unespecific) with at least 6 months of interval between smears. This cytological diagnosis was compared to histological diagnosis obtained by biopsy (large loop excision of the transformation zone) or cone biopsies, and considered negative when colposcopy was satisfactory without lesions or, when unsatisfactory, no lesion was detected after at least one cytological and colposcopic follow-up. Results: among the 215 cases, 49.3\% had negative results (CI 95\%: 42.6-55.9). The prevalence of histological confirmed low-grade squamous intraepithelial lesion was 38.6\% (CI 95\%: 32.1-45.1) and HSIL was 10.7\% (CI 95\%: 6.5-14.8). Cases of cancer were found in 1.4\% of patients (CI $95 \%: 0-2.9)$. We could not find a significant difference between the prevalence of HSIL/cancer according to age group using the cutoff point of 35 years. Conclusion: HSIL/cancer prevalence observed in this study has shown the risk of finding this kind of lesions in about $12 \%$ of women assisted in our public health system with two cytological diagnosis of ASCUS. A higher probability of HSIL/cancer in the different age groups was not found but this result was limited by our small sample size.

KEYWORDS: Atypical squamous cells; Cervical intraepithelial neoplasia; Cervix neoplasms; High-grade squamous intraepithelial lesions; Cervical cancer; Colposcopy; Cytodiagnosis

\footnotetext{
Trabalho desenvolvido no Instituto Fernandes Figueira (IFF)- FIOCRUZ- Rio de Janeiro- RJ- Brasil.

Mestranda em Saúde da Mulher - IFF- FIOCRUZ- Rio de Janeiro (RJ)- Brasil

Médico colposcopista, Doutor em Pesquisa Clinica e Mestre em Saúde da Mulher- IFF- FIOCRUZ- Rio de Janeiro (RJ)- Brasil

Médica colposcopista, Doutora em Saúde da Mulher e Mestre em Ginecologia - IFF- FIOCRUZ- Rio de Janeiro (RJ)- Brasil

Médica colposcopista e Mestranda em Saúde da Mulher - IFF- FIOCRUZ- Rio de Janeiro (RJ)- Brasil

Professor Titular de Obstetrícia da Faculdade de Medicina de Campos - Campos (RJ)- Brasil

6 Médica patologista do Departamento de Anatomia Patológica- IFF- FIOCRUZ- Rio de Janeiro (RJ)- Brasil

Correspondência: Fernanda Rangel da Veiga

Av. Raimundo Magalhães Junior 300, bloco 2, apto 102- Barra da Tijuca - 22793-050 - Rio de Janeiro - RJ - Tel: 8741-8876 - Fax: (21) 3328-2338 -

e-mail: frveiga@globo.com 


\section{Introdução}

Estima-se que no Brasil o câncer de colo uterino seja a terceira neoplasia maligna mais comum entre as mulheres, sendo, entre elas, a quarta causa de morte por câncer. A principal estratégia utilizada para rastreamento desta neoplasia e de suas lesões precursoras é o exame citopatológico do colo uterino ${ }^{1}$.

Para uniformizar o sistema de terminologias para este método diagnóstico, foi criado o Sistema Bethesda numa reunião de especialistas realizada na cidade de mesmo nome em Maryland, EUA, no ano de $1988^{2}$. Seu objetivo era estabelecer normas de classificação citológica para reduzir confusões diagnósticas entre alterações celulares benignas e realmente atípicas ${ }^{3}$. Nesta reunião foram introduzidos os termos citológicos de lesão intra-epitelial de baixo grau (low grade intraepithelial lesion - LSIL ou LIBG) compreendendo as alterações sugestivas de infecção pelo HPV e neoplasias intra-epiteliais de grau I (NIC I), lesão intra-epitelial de alto grau (high-grade intraepithelial lesion-HSIL ou LIAG), como expressões citológicas de NIC II e III, e atipias em células escamosas de significado indeterminado (atypical squamous cells of undetermined significance - ASCUS), definidas pela presença de achados citológicos insuficientes para o diagnóstico de lesão intraepitelial. Esta nova categoria, no entanto, apresenta limitações, pois não define se as alterações presentes à citologia são reparativas ou neoplásicas ${ }^{2}$.

Por este motivo foi revista a classificação citológica de ASCUS na última reunião realizada entre especialistas em Bethesda em 2001. Esta categoria foi reclassificada em "ASC-US" - "células escamosas atípicas, de significado indeterminado" - e "ASC-H" - "células escamosas atípicas não se podendo excluir lesão intra-epitelial de alto grau" ". O objetivo era diferenciar os casos em que há maior probabilidade de existir lesão precursora do câncer de colo uterino e que, por este motivo, devem ser encaminhados imediatamente para colposcopia. Apesar de esta proposta de subclassificação do que anteriormente se denominava ASCUS sem qualquer especificação ter sido discutida e adotada pela Sociedade Brasileira de Citopatologia a partir de $2002^{5}$, seu uso ainda não é observado pela maioria dos clínicos nem foi ainda adotado pelo programa governamental de prevenção do câncer de colo uterino (Viva Mulher) ${ }^{6}$. Em conseqüência, continuam as dúvidas quanto à necessidade de encaminhamento imediato destes casos para colposcopia. Vários trabalhos relacionam esta categoria citológica à presença de lesões intra-epiteliais ou carcinoma de colo uterino em porcentagens variáveis ${ }^{7,8}$.

O primeiro objetivo deste estudo é estimar a prevalência de LIAG e câncer em mulheres com citologia com diagnóstico de ASCUS persistente após 6 meses. Outro objetivo é verificar se a idade é fator indicador para a existência destas lesões neste grupo de mulheres, o que poderia indicar uma faixa etária que deveria ser priorizada no encaminhamento à colposcopia nos casos de diagnóstico citológico de ASCUS.

\section{Métodos}

Foram incluídas 215 mulheres residentes na região metropolitana do Rio de Janeiro, atendidas pelos postos municipais de saúde e referidas ao Instituto Fernandes Figueira da Fundação Oswaldo Cruz (IFF-FIOCRUZ), no período de janeiro de 1998 a agosto de 2005, com diagnóstico de ASCUS (sem especificação) persistente em duas citologias com intervalo mínimo de 6 meses. Todas estas mulheres estão nos grupos de baixa renda. Foram excluidas as gestantes e as com sorologia positiva para HIV. A média de idade foi de 36 anos.

Rotineiramente, recebemos em nosso serviço as pacientes pré-agendadas pela Secretaria Municipal de Saúde do Rio de Janeiro com citologias alteradas para colposcopia, segundo as recomendações do Programa Viva Mulher ${ }^{6}$. No caso de diagnóstico citológico de ASCUS, as pacientes são agendadas após o segundo exame com o mesmo resultado ou mais grave. Todas estas citologias são colhidas em postos municipais de saúde e enviadas a um laboratório central de grande porte, o Sistema Integrado Tecnológico em Citopatologia (SITEC), vinculado ao Instituto Nacional do Câncer. Este é considerado padrão de qualidade para o setor público no estado do Rio de Janeiro, sendo o laboratório responsável por todos os exames citológicos do sistema público da cidade do Rio de Janeiro e de vários outros municipios do Estado. No SITEC, todas as lâminas de citologia são analisadas por citotécnicos, sendo os casos positivos ou duvidosos para neoplasia encaminhados a médicos citopatologistas para revisão. Caso haja concordância para os casos positivos, estas lâminas são analisadas por um segundo citopatologista, definindo-se o diagnóstico citológico. Ainda como monitoramento interno de qualidade, $10 \%$ dos resultados negativos analisados por citotécnicos são encaminhados a citopatologistas para revisão.

Ao receber estas mulheres a abordagem é estabelecida após realização da colposcopia. Todos os exames foram realizados por médicos treinados sob supervisão de um colposcopista experiente, sendo utilizados o ácido acético a $2 \%$ e solução de Lugol. Fator fundamental a ser considerado no diagnóstico colposcópico é a visualização total ou não da zona de transformação ${ }^{9}$ do processo de substituição do epitélio. Quando o exame de toda a zona 
de transformação foi possivel, consideramos o exame satisfatório. Quando isto não foi possivel em um primeiro exame, costumamos rever a paciente após preparo estrogênico 9 . Quando a colposcopia foi satisfatória mas sem alterações colposcópicas, consideramo-la negativa. Quando satisfatória com alterações epiteliais, o diagnóstico final é dado pela biópsia. Quando é insatisfatória, consideramos o diagnóstico de alguma alteração observada e biopsiada e, quando não são vistas alterações colposcópicas em exame insatisfatório, consideramos o diagnóstico limitado e mantemos a paciente em seguimento citocolposcópico até que se obtenha diagnóstico positivo ou, após um ano, quando não há mais suspeita citológica.

A biópsia dirigida foi realizada quando presente qualquer alteração colposcópica sugestiva de lesão intra-epitelial ou câncer, como o epitélio aceto-reator e/ou iodo negativo à solução de Lugol, pontilhados, mosaicos ou vasos atípicos, conforme terminologia determinada pela Federação Internacional de Patologia Cervical e Colposcopia em Barcelona/2002 ${ }^{10}$.

Utilizamos para biópsias aparelho eletrocirúrgico de alta freqüência com freqüência de saída de $480 \mathrm{kHz}$ e potência utilizada de até $40 \%$ da máxima $(200 \mathrm{~W})$ com eletrodos em alça circular de aço inoxidável de $0,1 \mathrm{~mm}$ de espessura e $5 \mathrm{~mm}$ de diâmetro.

De acordo com o diagnóstico histopatológico destas biópsias, as LIAG foram tratadas pela exérese da zona de transformação (large loop excision of the transformation zone-LLETZ), nos casos em que a colposcopia foi satisfatória, ou conização do colo uterino, quando a colposcopia foi insatisfatória, sendo considerado conclusivo o diagnóstico histológico mais grave. As exéreses da zona de transformação e conizações foram realizadas com o mesmo aparelho de alta freqüência com alças semicirculares de filamento de aço inoxidável com $0,1 \mathrm{~mm}$ de espessura com $20 \times 10$ $\mathrm{mm}$ ou $15 \times 10 \mathrm{~mm}$ para LLETZ e, em casos de conizações, com variações de comprimento conforme cervicometria realizada imediatamente antes do procedimento cirúrgico. Nestes casos a potência utilizada no aparelho foi de até $60 \%$ da máxima. Também foram utilizados eletrodos retos em algumas conizações. As pacientes com LIBG foram mantidas em seguimento. Nestes casos, o resultado da biópsia foi considerado conclusivo para as LIBG ou para resultados negativos (inflamatórios, metaplasia escamosa, hiperplasia da camada basal).

Todos os espécimes de biópsias, LLETZ ou conizações foram encaminhados ao laboratório de Anatomia Patológica do Instituto Fernandes Figueira e analisados por médicos patologistas do IFF, com larga experiência em patologia cervical uterina.

Também foi considerado conclusivo o diagnóstico colposcópico negativo quando não havia lesão ou quando sugestivo de infecção pelo HPV sem NIC em colposcopias satisfatórias. Nas colposco- pias insatisfatórias sem lesão visivel, foi considerado diagnóstico negativo conclusivo se pelo menos um seguimento citológico e colposcópico foram negativos. Todos estes procedimentos estão incluídos nas rotinas de trabalho.

Para estabelecer a prevalência de lesões, calculamos a freqüência de diagnósticos com seus respectivos intervalos de confiança a 95\% (IC 95\%). Para a análise estratificada da distribuição de lesões de alto grau e câncer em função da faixa etária, optamos pelo ponto de corte de 35 anos por estar entre a média e a mediana de nossa amostra. Esta análise foi realizada por estarmos cientes de maior prevalência de lesões precursoras e câncer de colo uterino em pacientes de idade mais avançada ${ }^{11}$. Assim, optamos por utilizar ponto de corte que dividisse a amostra em duas faixas etárias em um ponto central. Para análise estatística da diferença de proporções de LIAG ou câncer em cada faixa de idade, foi utilizado teste do $\chi^{2}$, e ainda estimamos o risco destas lesões entre mulheres com mais de 35 anos pela razão de prevalências com seu IC 95\%.

Os dados foram armazenados em banco de dados padrão Microsoft Access 97 e para análise foram utilizados os aplicativos SPSS versão 8.0 e Epi-Info versão 6.04.

Esta pesquisa foi aprovada pelo Comitê de Ética em Pesquisa do Instituto Fernandes Figueira, em 26 de julho de 2005.

\section{Resultados}

Os casos de ASCUS apresentaram média de idade de 36,1 anos (DP=13,2), mediana de 34 anos, conforme distribuição por faixa etária descrita na Tabela 1. A maioria dos casos com diagnóstico citológico persistente de ASCUS estavam nas faixas etárias de 21 a 30 anos e de 41 a 50 anos, correspondendo a 29,3 e $25,6 \%$ dos casos, respectivamente. As menores freqüências deste diagnóstico encontraramse nas faixas etárias de 61 a 70 anos, com 3,3\%, e acima de 71 anos, que corresponde a $0,9 \%$ dos casos.

Tabela 1 - Distribuição dos 215 casos com diagnóstico citológico de ASCUS por faixa etária.

\begin{tabular}{lcr}
\hline Idade & Freqüência & $\%$ \\
\hline até 20 anos & 23 & 10,7 \\
$21-30$ anos & 63 & 29,3 \\
$31-40$ anos & 44 & 20,5 \\
$41-50$ anos & 55 & 25,6 \\
$51-60$ anos & 21 & 9,8 \\
$61-70$ anos & 7 & 3,3 \\
$>70$ anos & 2 & 0,9 \\
Total & 215 & 100,0 \\
\hline
\end{tabular}

Na Tabela 2 está exposta a distribuição dos diagnósticos conclusivos. A prevalência de LIAG foi 
de 10,7\% (IC 95\%: 6,5-14,8) e de LIBG de 38,6\% (IC 95\%: $32,1-45,1)$. Casos de câncer foram encontrados em 1,4\% das pacientes (IC 95\%: 0-2,9) e 49,3\% dos casos (IC 95\%: 42,6-55,9) foram negativos.

Tabela 2 - Distribuição de diagnósticos definitivos em pacientes com diagnóstico citológico de ASCUS em duas amostras consecutivas.

\begin{tabular}{|c|c|c|c|}
\hline Diagnóstico definitivo & Freqüência & $\%$ & IC $95 \%$ \\
\hline Negativo & 106 & 49,3 & $42,6-55,9$ \\
\hline $\begin{array}{l}\text { Lesão intra-epitelial de baixo grau } \\
\text { - LIBG (HPV/NIC I) }\end{array}$ & 83 & 38,6 & $32,1-45,1$ \\
\hline $\begin{array}{l}\text { Lesão intra-epitelial de alto grau - } \\
\text { LIAG (NIC II/III) }\end{array}$ & 23 & 10,7 & $6,5-14,8$ \\
\hline Câncer & 3 & 1,4 & $0-2,9$ \\
\hline Total & 215 & 100,0 & \\
\hline
\end{tabular}

Conforme exposto na Tabela 3, em mulheres de até 35 anos (112 casos - 52,1\% da amostra) houve 10 casos (9\%) de LIAG/câncer. Entre aquelas com mais de 35 anos (103 casos - 47,9\%) houve 16 casos (15\%) do mesmo diagnóstico definitivo. Embora esta diferença de proporções não tenha sido significativa $\left(\chi^{2}=2,2\right.$ e $\left.p=0,14\right)$, a razão de prevalência de 1,7 (IC 95\%: 0,8-3,6), apesar de imprecisa, aponta a possibilidade de utilizar ponto de corte em função da idade para encaminhamento à colposcopia. A diferença não significativa pode ser apenas decorrente do tamanho amostral, faltando poder para confirmá-la (possível erro â ou tipo 2 , ou seja, afirmar que não há diferença entre as proporções quando ela realmen- te pode existir mas não foi possivel demonstrar devido à limitação do tamanho amostral).

Tabela 3 - Distribuição dos diagnósticos definitivos em mulheres recebidas com diagnóstico citológico persistente de ASCUS por faixa etária.

\begin{tabular}{lccc}
\hline Idade & NEG/LIBG & LIAG/Cancer & Total \\
\hline Até 35 anos & 102 & 10 & 112 \\
$>$ 35 anos & 87 & 16 & 103
\end{tabular}

\section{Discussão}

O diagnóstico citológico de ASCUS é bastante discutivel, mesmo na presença de rigorosos critérios para seu estabelecimento. Estudo realizado em Belo Horizonte mostrou discordância entre diferentes análises de lâminas com diagnóstico de ASCUS pelo mesmo citopatologista, o que demonstra subjetividade nos laudos citopatológicos desta categoria ${ }^{12}$. Tal fato leva alguns serviços a realizar revisão das citologias das pacientes encaminhadas com o diagnóstico de ASCUS. Em nosso serviço, todavia, não realizamos este procedimento, pois poderia acarretar atraso no estabelecimento de condutas e, possivelmente, perda de pacientes. Por outro lado, nossos dados refletem a prática rotineira dos pólos de colposcopia no município do Rio de Janeiro e a partir deles pode-se inferir a prevalência de LIAG e câncer em pacientes recebidas com diagnóstico de ASCUS fornecido pelo mesmo laboratório (SITEC).

No Quadro 1 apresentamos resumo dos estudos publicados que procuraram responder à mesma questão por nós estudada.

Quadro 1 - Estudos publicados sobre prevalência de lesão intra-epitelial de alto grau em citologias de ASCUS no período de 1994 a 2004

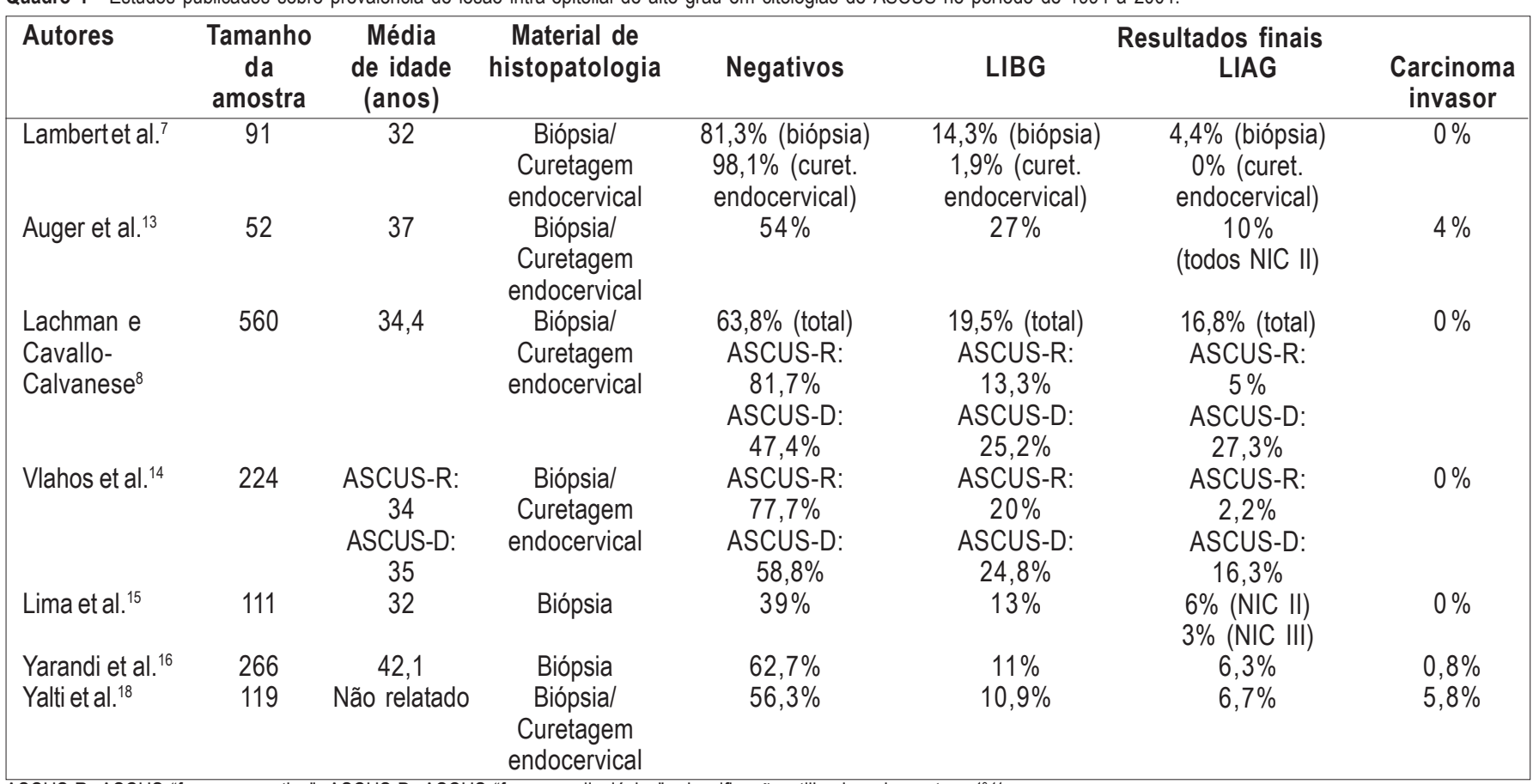


Observa-se que a prevalência de LIAG definida histologicamente após biópsias e curetagens endocervicais varia de 4,4 a 10\% dos $\operatorname{casos}^{7,13}$. Em um dos estudos a curetagem endocervical não foi capaz de demonstrar lesão precursora ou câncer em nenhum dos casos ${ }^{7}$. Além da LIAG pode-se encontrar carcinoma invasor em $4 \%$ dos casos.

Estudo realizado na Turquia, no qual foram incluídas 119 pacientes, descreve prevalência de $6,7 \%$ de LIAG e 5,8\% de câncer cervical ${ }^{8}$.

Em estudo de grande porte ${ }^{14}$ com 560 casos de citologias que receberam resultados de ASCUS e outro com amostra de 224 pacientes ${ }^{15}$, foi analisada a correlação cito-histológica empregando-se a nova classificação sugerida no encontro de Bethesda de 2001. Conforme mostra o Quadro 1, observou-se maior prevalência de lesão intra-epitelial (LIBG ou LIAG) nos grupos com citologia ASCH (ou ASCUS "não podendo excluir lesão intra-epitelial de alto grau") em relação ao grupo ASC-US (ou ASCUS "favorece reativo"), o que indica a utilidade desta subclassificação nestes casos.

Estudos nos quais se utiliza somente biópsia para resultados definitivos mostraram prevalências de LIAG de $6,3 \%{ }^{15}$ e $6 \%$ de NIC II e $3 \%$ de NIC III ${ }^{16}$. Neste último também foi encontrado câncer cervical em $0,8 \%$ dos 111 casos com diagnóstico citológico de ASCUS em pacientes do estado de Pernambuco.

É possivel observar que encontramos resultados diferentes daqueles descritos por alguns autores em termos de prevalência de lesões mais graves. Isto pode ser explicado por diferenças entre os estudos no que diz respeito ao método diagnóstico empregado e seleção da amostra, como discutido a seguir. Em nosso serviço não utilizamos a curetagem endocervical como método diagnóstico endocervical, pelas limitações que este método oferece em termos de qualidade do espécime obtido.

Outro fator a ser considerado é a utilização de amostras com somente um resultado citológico de ASCUS em todos os estudos analisados. Nossa amostra constitui-se de pacientes com duas citologias com diagnóstico persistente de ASCUS com intervalo mínimo de seis meses. Esta seleção aumenta a probabilidade de encontrarmos lesões intra-epiteliais, configurando viés de seleção mas que reflete a prática dos pólos de colposcopia que seguem as recomendações do Programa Viva Mulher.

Além disso, não consideramos somente a biópsia para resultados definitivos. Levamos em consideração todos os procedimentos de tratamento, como as exéreses da zona de transformação e conizações, considerando sempre o diagnóstico histológico mais grave.

Nosso estudo ainda incluiu casos de colposcopia insatisfatória sem lesão visível, considerando pelo menos uma citologia e colposcopia no se- guimento com intervalo mínimo de 3 meses para considerá-los negativos, o que torna mais confiável o diagnóstico nestes casos.

Nossa amostra foi suficiente para trazer resultados importantes para a nossa população, podendo orientar os clínicos de nosso meio na tomada de decisão de encaminhar à colposcopia. A prevalência relativamente baixa (cerca de $10 \%$ ) de casos de lesões precursoras, aqui entendidas como aquelas com maior probabilidade de progressão para o câncer caso não tratadas (LIAG - NIC II/ III), mostra que a conduta conservadora de encaminhar a paciente para colposcopia somente após duas citologias com diagnóstico de ASCUS apenas retarda seu diagnóstico em seis meses, o que não parece agravar seu prognóstico. Também reforça a menor importância deste diagnóstico citológico a baixa prevalência de câncer que encontramos em nossa amostra (1,4\%). Esta estratégia é justificada para priorização dos recursos de colposcopia para pacientes com maior probabilidade de terem uma LIAG ou câncer, isto é, pacientes com citologias sugestivas destas lesões.

A análise do risco de ter LIAG/câncer considerando o corte de idade em 35 anos não foi capaz de demonstrar que este pode ser um delimitador de condutas, mas pode ter sido limitada por pequeno tamanho amostral, merecendo investigação com maior número de pacientes. Existem dados na literatura que relacionam menor possibilidade de lesões precursoras e câncer em mulheres com diagnóstico citológico de ASCUS na pós-menopausa ${ }^{17}$. Isto se deve ao fato de que características inerentes ao hipoestrogenismo podem acarretar dificuldades na interpretação citológica, inclusive algumas alterações citológicas podem ser indistinguiveis da classificação de ASCUS. Processos inflamatórios e degenerativos associados à vaginite atrófica podem resultar em alterações celulares falsamente interpretadas como ASCUS, ou até mesmo lesões mais graves ${ }^{17}$.

Esperamos que a nova classificação na qual se discriminem os casos de ASCUS possivelmente neoplásicos comece a ser utilizada de forma mais consistente, para que em análise posterior, possamos verificar sua utilidade em nosso meio.

Concluímos que a prevalência de lesões precursoras e câncer cervical encontrada em nosso estudo mostra que o risco de encontrarmos este tipo de lesão em mulheres com duas citologias com diagnóstico de ASCUS com intervalo de seis meses é de cerca de $12 \%$. Nossa análise por faixa etária não foi capaz de mostrar diferença significativa entre a distribuição dos casos de LIAG ou câncer em mulheres de até 35 anos ou com mais de 35 anos, não sendo possivel recomendar este fator como discriminante para encaminhamento à colposcopia. 
Não existe orçamento financeiro para esta pesquisa. Os procedimentos que são abordados neste estudo fazem parte da rotina do Setor de Patologia Cervical - Departamento de Ginecologia - IFF. Os pesquisadores não foram remunerados.

\section{Referências}

1. Ministério da Saúde. Instituto Nacional do Câncer [homepage da Internet]. Câncer do colo do útero. 2005 [citado 2005 Jun 7]. Disponível em: http:// www.inca.gov.br/conteudo_view.asp?id=326.

2. The 1988 Bethesda System for reporting cervical/ vaginal cytological diagnosis. National Cancer Institute Workshop. JAMA. 1989;262(7):931-4.

3. Pedrosa ML, Mattos IE, Koifman OJ, Silva RJO, Athayde MJPM. Atipias escamosas de significado indeterminado: uma revisão da literatura. DST J Bras Doenças Sex Transm. 2003;15(3):46-51.

4. Solomon D, Davey D, Kurman R, Moriarty A, O'Connor D, Prey M, et al. The 2001 Bethesda System: terminology for reporting results of cervical cytology. JAMA. 2002;287(16):2114-9.

5. Sociedade Brasileira de Citopatologia [homepage da Internet]. Nomenclatura brasileira para laudos de citopatologia cervical uterina. 2001 [citado 2004 Nov 24]. Disponivel em: http://www.citopatologia.org.br/ nclatura.htm.

6. Ministério da Saúde. Instituto Nacional do Câncer [homepage da Internet]. Nomenclatura brasileira para laudos citopatológicos cervicais e condutas clínicas preconizadas. 2002 [citado 2003 Mar 1]. Disponivel em: http://www.inca.gov.br/viva_mulher.

7. Lambert B, Boivin Y, Lepage Y. Atypical squamous cells of undetermined significance (ASCUS): clinicalcytological significance. Int $\mathrm{J}$ Gynecol Cancer. 1999;9(4):329-32.

8. Lachman MF, Cavallo-Calvanese C. Qualification of atypical squamous cells of undetermined significance in an independent laboratory: is it useful or significant? Am J Obstet Gynecol. 1998;179(2):421-9.

9. Cartier R, Cartier I. Colposcopia prática. $3^{\mathrm{a}}$ ed. São Paulo: Roca; 1994.

10.Walker P, Dexeus S, De Palo G, Barrasso R, Campion $M$, Girardi $F$, et al. International terminology of colposcopy: an updated report of the International Federation for Cervical Pathology and Colposcopy. Obst Gynecol. 2003;101(1):175-7.

11. Ministério da Saúde. Instituto Nacional do Câncer [homepage da Internet]. Morbi-mortalidade por câncer do colo do útero. 2002 [citado 2005 Jun 7]. Disponivel em: http://www.inca.gov.br/ viva_mulher/manual/documentos/morbi.pdf.

12. Souza JHK, Kalil IV, Leite JM, Geber S. Avaliação de lâminas de colpocitologia oncótica previamente diagnosticadas como ASCUS: comparação interensaio e interobservadores. Rev Bras Ginecol Obstet. 2004;26(3):233-40.

13. Auger M, Charbonneau M, Arseneau J. Atypical squamous cells of undetermined significance. A cytohistologic study of 52 cases. Acta Cytol. 1997;41(6):1671-5.

14. Vlahos NP, Dragisic KG, Wallach EE, Burroughs FH, Fluck S, Rosenthal DL. Clinical significance of the qualification of atypical squamous cells of undetermined significance: an analysis on the basis of histologic diagnoses. Am J Obstet Gynecol. 2000;182(4):885-90.

15. Lima DNO, Câmara S, Mattos MGG, Ramalho R. Diagnóstico citológico de ASCUS: sua importância na conduta clínica. J Bras Patol Med Lab. 2002;38(1):45-9.

16. Yarandi F, Izadi Mood N, Mirashrafi F, Eftekhar Z. Colposcopic and histologic findings in women with a cytologic diagnosis of atypical squamous cells of undetermined significance. Aust N Z J Obstet Gynaecol. 2004;44(6):514-6.

17. Flynn K, Rimm DL. Diagnosis of "ASCUS" in women over age 50 is less likely to be associated with dysplasia. Diagn Cytopathol. 2001;24(2):132-6.

18. Yalti S, Gurbuz B, Bilgic R, Cakar Y, Eren S. Evaluation of cytologic screening results of the cervix. Int J Gynecol Cancer. 2005;15(2):292-4. 